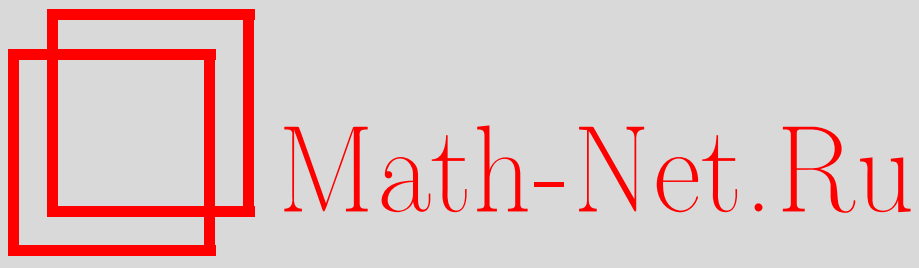

А. Х. Бикулов, А. П. Зубарев, Л. В. Кайдалова, Иерархическая динамическая модель финансового рынка вблизи точки обвала и $p$-адический математический анализ, Вестн. Сам. гос. техн. ун-та. Сер. Физ.-мат. науки, 2006, выпуск 42, 135140

DOI: https://doi.org/10.14498/vsgtu424

Использование Общероссийского математического портала Math-Net.Ru подразумевает, что вы прочитали и согласны с пользовательским соглашением

http://www.mathnet.ru/rus/agreement

Параметры загрузки:

IP: 54.81 .137 .203

26 апреля 2023 г., 13:51:13

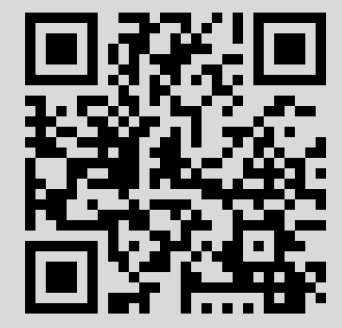




\title{
ИЕРАРХИЧЕСКАЯ ДИНАМИЧЕСКАЯ МОДЕЛЬ ФИНАНСОВОГО РЫНКА ВБЛИЗИ ТОЧКИ ОБВАЛА И Р-АДИЧЕСКИЙ МАТЕМАТИЧЕСКИЙ АНАЛИЗ
}

\begin{abstract}
В серии работ ряда зарубежных авторов, посвященных математическому исследованию финансовых рынков, было обнаружено, что поведение логарифмов цен на финансовых рынках вблизи точек обвала имеет вид степенного закона, умноженного на сумму логопериодических гармоник. В данной статье предлагается простая микроскопическая иерархическая модель финансового рынка на временах, предшествующих моменту обвала рынка. В рамках смоделированного взаимодействия между участниками рынка описывается динамика данной модели уравнением Чемпена-Колмогорова и показывается, что это уравнение эквивалентно модифицированному уравнению Владимирова, известному из р-адического анализа. При больших временах, предиествующих моменту обвала рын$\kappa a$, эта модель производит степенной закон, умноженный на сумму логопериодических гармоник для изменения иеены актива.
\end{abstract}

Введение. В колебаниях курсов ценных бумаг на финансовых рынках проявляется ряд закономерностей, которые пока еще не могут быть описаны в рамках какой-либо модели. Одной из этих закономерностей является тот факт, что на больших временных масштабах усредненные логарифмы цен имеют тенденцию степенного роста со временем с резкими спадами, соответствующими обвалам рынка (финансовым крахам).

В последнее десятилетие было предложено рассматривать фондовые рынки как сложные динамические неравновесные системы, изучаемые в физике, для описания которых используется микроскопический подход [1]. Переход от микроскопических масштабов к макроскопическим обычно является сложной задачей, которая в свое время была исследована в одной из областей теоретической физики - квантовой статистике. Основной концепцией этого перехода является понятие масштабной инвариантности. Следствием масштабной инвариантности системы является то, что ее динамика описывается самоподобными функциями. Кроме того, оказывается, что каждая система, характеризуемая некоторым типом масштабной инвариантности, имеет критическую точку, около которой ее поведение развивается необычным образом. По мнению авторов монографии [1] в финансовых системах крах может рассматриваться именно как критическая точка, и ее наличие связано именно с законом подобия финансовой системы так называемой дискретной масштабной инвариантностью. Одним из важнейших следствий дискретной масштабной инвариантности является степенной закон поведения динамической системы, умноженный на сумму логопериодических гармоник. Поясним это на простом примеpe.

Рассмотрим некоторую наблюдаемую величину $O(t)$ ( $t$ - некоторый параметр, не обязательно время). Говорят, что система масштабно инвариантна, если любая наблюдаемая величина удовлетворяет соотношению

$$
O(t)=\mu(\lambda) O(\lambda t)
$$

для любого $\lambda$ с некоторой функцией $\mu(\lambda)$. Дискретная масштабная инвариантность означает, что условие (1) имеет силу не для всех $\lambda$, а только для дискретного набора $\lambda_{n}=v^{n}$, где $v-$ некоторое число и $n=1,2,3, \ldots$ В этом случае общим решением уравнения (1) является функция

$$
O(t)=A t^{\alpha} P(\ln t / \ln v),
$$

где $P(x)$ - периодическая функция с периодом 1. Разлагая $P(x)$ в ряд Фурье, получим степенной закон, умноженный на сумму логопериодических гармоник:

$$
O(t)=A t^{\alpha} \sum_{n=-\infty}^{\infty} C_{n} \cos \left(2 \pi n \ln t / \ln v-\phi_{n}\right) .
$$

В начале 90-х годов в ряде работ было показано, что изменение параметров некоторых сложных физических систем вблизи критической точки описывается законом, подобным закону (2) (см. ссылки в [1]). В серии работ, посвященных исследованию финансовых рынков путем анализа эмпирических данных, было обнаружено, что поведение логарифмов цен на финансовых рынках вблизи точки краха (обвала) рынка, действительно напоминает степенной закон, умноженный на сумму логопериодических гармоник [1]. Тем не менее, следует отметить, что на сегодняшний день не существует какой-либо микроскопической динамической модели, учи- 
тывающей взаимодействие участников рынка, в рамках которой закон (2) поведения цен вблизи точки краха был бы воспроизведен.

В работах [2,3] было отмечено, что дискретная масштабная инвариантность имеет место для иерархически организованных систем. Именно для таких систем характерно существование критической точки (времени $t_{c}$ ) в окрестности которой имеются степенное поведение, умноженное на логарифмические осцилляции.

В настоящей работе предлагается простая микроскопическая иерархическая модель финансового рынка, на временах, предшествующих моменту обвала рынка. Показано, что эта модель приводит к произведению степенного закона, умноженного на сумму логопериодических гармоник для изменения цены актива.

Иерархический подход к микроскопическому моделированию финансовых рынков и степенной закон поведения логарифмов цен вблизи точек краха. В данной работе описывается динамика финансового рынка вблизи точки его обвала, т. е. в течение некоторого временного интервала, предшествующего переходу рынка в новую фазу. Если в обычном состоянии рынка действия его участников (назовем их агентами) носят стихийный характер (различные агенты в каждый момент времени могут иметь противоположные позиции), то в момент обвала позиция всех участников рынка одинакова, иными словами все агенты одновременно принимают решение купить принадлежащие им активы. Начало обвала рынка можно представить себе следующим образом. Начиная с некоторого момента времени $t_{1}$, предшествующего моменту обвала рынка, действия части агентов начинают происходить в одинаковой фазе, причем число агентов, находящихся в этой фазе лавинообразно увеличивается с течением времени. Наша цель - построить динамику этого процесса при временах $t>t_{1}$ и до момента обвала, при котором рынок переходит в новую фазу.

Предположим, что любой рынок устроен иерархически. Участники рынка объединены в группы, это группы в свою очередь объединены в большие группы и так далее. В основу объединения участников рынка в группы может быть положена степень влияния агентов друг на друга, которая в количественном выражении может быть описана в терминах ультраметрического расстояния. Агенты, входящие в одну группу (имеющие одинаковый уровень иерархии) больше подвержены влиянию друг на друга, чем агенты, входящие в различные группы. Данную структуру рынка можно представить в виде ультраметрического дерева (см. рис., в котором $p=2)$. На этом дереве точки нижнего ряда отождествляются с агентами. Будем предполагать, что полное число агентов бесконечно велико $(L \rightarrow \infty)$.

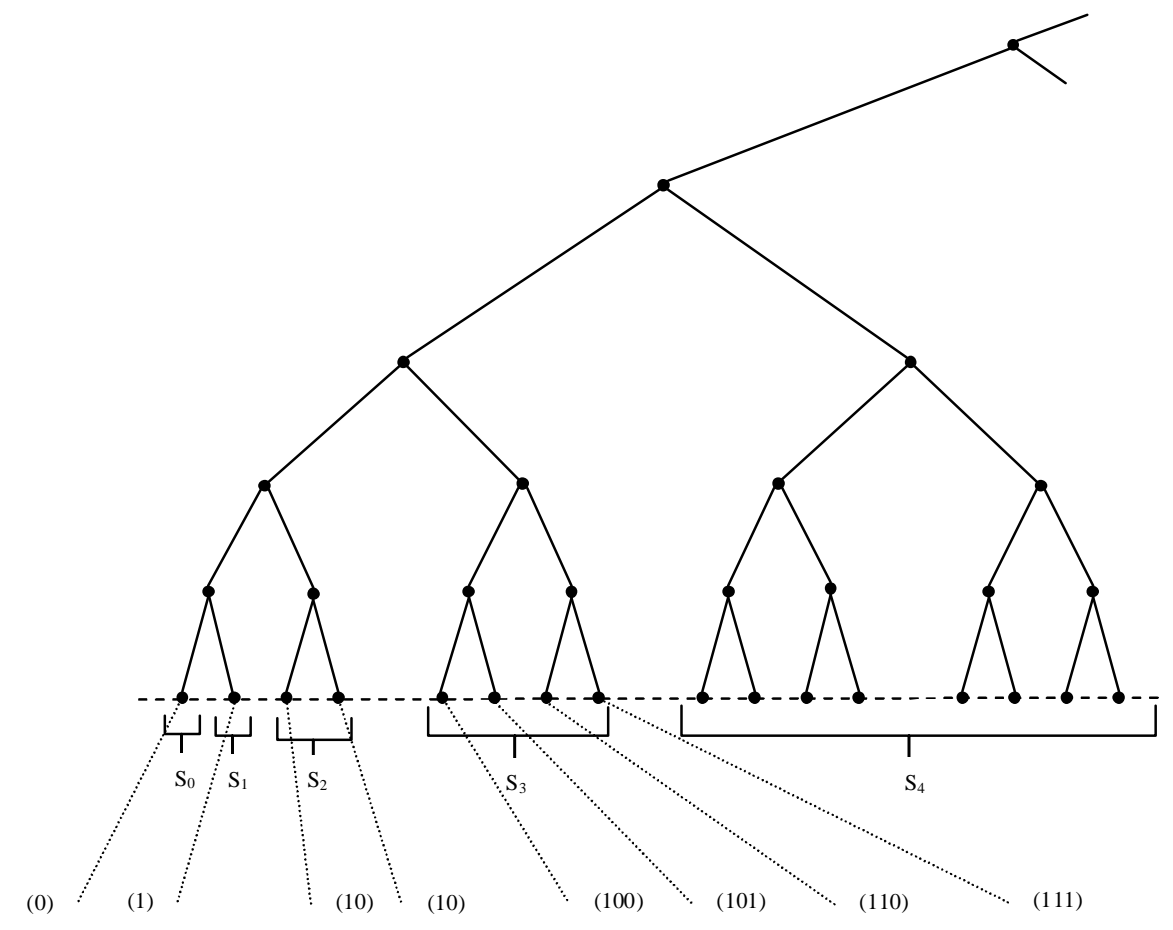

Регулярное ультраметрическое дерево, моделирующее иерархическую структуру множества участников финансового рынка 
На рисунке агенты соответствуют точкам на нижней стороне дерева. Для дальнейшего удобства индексации точек разобьем эти точки на множества $S_{0}, S_{1}, S_{2}, S_{3}, \ldots$ Множество $S_{0}$ состоит из одной точки, которой сопоставим индекс (0). Множество $S_{1}$ состоит из $p-1$ точки, которые будем обозначать индексом $\left(b_{1}\right), b_{1}=1, \ldots, p-1$. Множество $S_{2}$ состоит из $(p-1) p$ точек, которые будем обозначать индексом $\left(b_{1} b_{2}\right), b_{1}=0, \ldots, p-1, b_{2}=1, \ldots, p-1$, и так далее. Таким образом, каждую точку на дереве можно обозначить мультииндексом $\left(b_{1} b_{2} \ldots b_{k}\right)$ (см. рисунок).

Построенная ультраметрическая структура множества участников рынка позволяет ввести ультраметрическое расстояние между ними, соответствующее ультраметрическому расстоянию между соответствующими точками дерева. Это ультраметрическое расстояние, можно ввести различными способами. Имея ввиду связь между нижними точками ультраметрического дерева и множеством $p$-адических чисел [4], зададим ультраметрическое расстояние $d_{b_{1} b_{2} \ldots b_{k}, c_{1} c_{2} \ldots c_{l}}$ между агентами $\left(b_{1} b_{2} \ldots b_{k}\right)$ и $\left(c_{1} c_{2} \ldots c_{l}\right)$ следующим образом:

$$
d_{b_{1} b_{2} \ldots b_{k}, c_{1} c_{2} \ldots c_{l}}=p^{\max (k, l)} .
$$

Будем считать, что на точках $\left(b_{1} b_{2} \ldots b_{k}\right)$ задана функция $f_{b_{1} b_{2} \ldots b_{k}}(t)$, которая имеет смысл вероятности принятия агентом $\left(b_{1} b_{2} \ldots b_{k}\right)$ положительного решения относительно покупки финансовых активов. Таким образом, $0 \leq f_{b_{1} b_{2} \ldots b_{k}}(t) \leq 1$. В данной модели также вводится один агент (будем для определенности считать, что он соответствует точке (0)), который в любой момент времени имеет положительное решение о покупке активов. Будем также считать, что в начальный момент времени $t=0$ все агенты, отличные от агента $(0)$, имеют отрицательное решение о покупке. Таким образом, агент (0) будет влиять на других участников рынка, и в последующие моменты времени у других участников рынка будет увеличиваться вероятность принятия ими положительного решения. Это увеличение также будет происходить вследствие взаимодействия участников рынка между собой, которое задается ультраметрически с учетом иерархической структуры рынка. С математической точки зрения агент (0) с вероятностью $f_{0}(t)=1$ играет роль внешнего источника: он влияет на других агентов, но они на него не оказывают влияния.

Будем считать, что динамики вероятности принятия решения агентами задается уравнением Чемпена-Колмогорова

$$
\begin{gathered}
\frac{d f_{b_{1} b_{2} \ldots b_{k}}(t)}{d t}=\sum_{l=1}^{\infty} \sum_{c_{1}=1}^{p} \sum_{c_{2}=1}^{p} \ldots \sum_{c_{k}=1}^{p}\left(w_{b_{1} b_{2} \ldots b_{k}, c_{1} c_{2} \ldots c_{l}} f_{c_{1} c_{2} \ldots c_{l}}(t)-w_{b_{1} b_{2} \ldots b_{k}, c_{1} c_{2} \ldots c_{l}} f_{b_{1} b_{2} \ldots b_{l}}(t)\right)+ \\
+w_{b_{1} b_{2} \ldots b_{k}, 0} f_{0}(t)-w_{b_{1} b_{2} \ldots b_{k}, 0} f_{b_{1} b_{2} \ldots b_{l}}, \\
\frac{d f_{0}(t)}{d t}=0,
\end{gathered}
$$

где $w_{b_{1} b_{2} \ldots b_{k}, c_{1} c_{2} \ldots c_{l}}-$ вероятность перехода между агентами $\left(b_{1} b_{2} \ldots b_{k}\right)$ и $\left(c_{1} c_{2} \ldots c_{l}\right)$. Вероятность $w_{b_{1} b_{2} \ldots b_{k}, c_{1} c_{2} \ldots c_{l}}$ зададим в виде простой функции ультраметрического расстояния между агентами высшего уровня иерархии:

$$
w_{b_{1} b_{2} \ldots b_{k}, c_{1} c_{2} \ldots c_{l}}=\left(d_{b_{1} b_{2} \ldots b_{k}, c_{1} c_{2} \ldots c_{l}}\right)^{-\alpha-1}=p^{-\max (k, l)(\alpha+1)},
$$

где $\alpha>1$. Разумеется, возможен и другой выбор для $w_{b_{1} b_{2} \ldots b_{k}, c_{1} c_{2} \ldots c_{l}}$. Наш выбор замечателен тем, что он приводит к уравнению со структурой уравнения Владимирова [4-7] для функции вероятности, заданной на поле $p$-адических чисел, для которого возможно получить точное решение. Кроме того, потребуем $f_{0}(t)=1, f_{b_{1} b_{2} \ldots b_{k}}(0)=0$ для всех $\left(b_{1} b_{2} \ldots b_{k}\right) \neq(0)$. Тогда основное кинетическое уравнение можно переписать в виде

$$
\begin{aligned}
\frac{d f_{b_{1} b_{2} \ldots b_{k}}(t)}{d t}=\sum_{l=1}^{\infty} \sum_{c_{1}=1}^{p} \sum_{c_{2}=1}^{p} \ldots & \sum_{c_{1}=1}^{p}\left(p^{-\max (k, l)(\alpha+1)} f_{c_{1} c_{2} \ldots c_{l}}(t)-p^{-\max (k, l)(\alpha+1)} f_{b_{1} b_{2} \ldots b_{l}}(t)\right)+ \\
& +p^{-k(\alpha+1)}-p^{-k(\alpha+1)} f_{b_{1} b_{2} \ldots b_{l}}(t) .
\end{aligned}
$$

Решение бесконечной системы уравнений (3) представляет определенные трудности. Поэтому преобразуем систему (3) к уравнению для функции, заданной на поле $p$-адических чисел [4]. С этой целью сопоставим каждой точке нижнего края дерева определенные подмножества 
множества $p$-адических чисел $Q_{p}$ следующим образом. Точку $S_{0}$ на дереве представим множеством $p$-адических чисел

$$
S_{0}=Z_{p}=\left\{a_{0}+a_{1} p+a_{2} p^{2}+a_{3} p^{3}+\ldots\right\}
$$

где $a_{i}=0, \ldots, p-1, i=1, \ldots, \infty$. Далее, $p-1$ точек $S_{1}$ представим множеством

$$
S_{1}=\left\{b_{1} p^{-1}+a_{0}+a_{1} p+a_{2} p^{2}+a_{3} p^{3}+\ldots\right\}=\left\{b_{1} p^{-1}+Z_{p}\right\}
$$

где $b_{1}=1, \ldots, p-1$. Затем $(p-1) p$ точек $S_{2}$ представим множеством

$$
S_{2}=\left\{b_{2} p^{-2}+b_{1} p^{-1}+a_{0}+a_{1} p+a_{2} p^{2}+a_{3} p^{3}+\ldots\right\}=\left\{b_{2} p^{-2}+b_{1} p^{-1}+Z_{p}\right\},
$$

где $b_{2}=1, \ldots, p-1$ и $b_{1}=0, \ldots, p-1$, и так далее. Все множество точек $M$ нижнего края дерева представляет собой фактор множество $M=Q_{p} / Z_{p}$. Например, $(0) \equiv Z_{p}, \quad\left(b_{1}\right) \equiv b_{1} p^{-1}+Z_{p}$, $\left(b_{1} b_{2}\right) \equiv b_{2} p^{-2}+b_{1} p^{-1}+Z_{p}$ и так далее.

Строго говоря, для данной конструкции нужно считать $p$ простым числом. Тем не менее, полученные в дальнейшем результаты будут справедливы и для случая, когда $p$ - любое целое положительное число, большее 1.

Функция $f_{b_{1} b_{2} \ldots b_{k}}(t)$ становится функцией на $Q_{p} / Z_{p}$. Будем считать ее функцией на $Q_{p}$, т. е. $f_{b_{1} b_{2} \ldots b_{k}}(t)=f(x, t), x \in Q_{p}$, подразумевая ее постоянство на классах смежности $Q_{p} / Z_{p}$. Более того, функция $f(x, t)$ в силу выбранных начальных условий будет постоянна на всех $S_{i}$, $i=0,1, \ldots, \infty$. Тогда основное кинетическое уравнение уравнения можно записать в виде

$$
\begin{gathered}
\frac{d f(x, t)}{d t}=w_{0} \bar{\Omega}\left(|x|_{p}\right) \int_{Q_{p}} \frac{f(y, t)-f(x, t)}{|x-y|_{p}^{\alpha+1}} d_{p} y, \\
f(x, t)=1, \quad x \in Z_{p},
\end{gathered}
$$

где

$$
\bar{\Omega}\left(|x|_{p}\right)=1-\Omega\left(|x|_{p}\right), \quad \Omega\left(|x|_{p}\right)= \begin{cases}1, & |x|_{p} \leq 1, \\ 0, & |x|_{p}>1,\end{cases}
$$

а интеграл в (4) берется по множеству $Q_{p}$ по мере Хаара $d_{p} y$. Хотя интеграл берется по всему $Q_{p}$, а не по $M=Q_{p} / Z_{p}$, уравнение (4) совпадает с уравнением (3), поскольку функция $f(x, t)$ постоянна на классах смежности $x Z_{p}$, где $x \in M=Q_{p} / Z_{p}$, а мера множества $Z_{p}$ равна 1.

Уравнение (4) есть не что иное, как модифицированное уравнение Владимирова, известное из $p$-адического анализа [4-7].

Величину $s(t)=\int_{Q_{p}} f(x, t) d_{p} x$ можно интерпретировать как среднее число агентов, принявших к моменту времени $t$ положительное решение о покупке активов. Мы принимаем естественную гипотезу, что относительное изменение цены актива $P(t)$ пропорционально изменению среднего числа агентов, принявших положительное решение, т. е.

$$
\frac{d P(t)}{P(t)}=\mu d s(t)
$$

откуда следует

$$
\ln P(t)=\mu s(t) \text {. }
$$

Для нахождения $s(t)$ решим основное кинетическое уравнение. С этой целью сделаем замену

$$
f(x, t)=\Omega\left(|x|_{p}\right)+\bar{\Omega}\left(|x|_{p}\right) g(x, t)
$$

Тогда уравнение примет вид

$$
\begin{gathered}
\bar{\Omega}\left(|x|_{p}\right) \frac{d g(x, t)}{d t}=w_{0} \bar{\Omega}\left(|x|_{p}\right) \Gamma_{p}(-\alpha) D^{\alpha}\left[\bar{\Omega}\left(|x|_{p}\right) g(x, t)\right]+w_{0} \bar{\Omega}\left(|x|_{p}\right)|x|_{p}^{-\alpha-1}, \\
g(x, 0)=0,|x|_{p}>1,
\end{gathered}
$$


где $D^{\alpha}$ - оператор Владимирова, $\Gamma_{p}(-\alpha)=\frac{1-p^{-\alpha-1}}{1-p^{\alpha}}-p$-адическая гамма-функция [4]. К функции $\varphi(x, t)=\bar{\Omega}\left(|x|_{p}\right) g(x, t)$ можно применить $p$-адическое преобразование Фурье

$$
\varphi(x, t)=\bar{\Omega}\left(|x|_{p}\right) g(x, t)=\int_{Q_{p}} \tilde{\varphi}(k, t) \chi(-k x) d_{p} k,
$$

где $\chi(-k x)$ - характер поля $Q_{p}[4]$. После ряда преобразований, уравнение (6) приводится к виду

$$
\begin{gathered}
\bar{\Omega}\left(|x|_{p}\right) \int_{Q_{p}} \frac{d \tilde{\varphi}(k, t)}{d t} \chi(-k x) d_{p} k=w_{0} \bar{\Omega}\left(|x|_{p}\right) \Gamma_{p}(-\alpha)\left[\int_{Q_{p}}|k|_{p}^{\alpha} \tilde{\varphi}(k, t) \chi(-k x) d_{p} k\right]+ \\
+w_{0} \bar{\Omega}\left(|x|_{p}\right) \int_{Q_{p}} \Omega\left(|k|_{p}\right)\left[\frac{1-p^{-1}}{p^{\alpha}-1}-\Gamma_{p}(-\alpha)|k|_{p}^{\alpha}\right] \chi(-k x) d_{p} k .
\end{gathered}
$$

Данное уравнение выполнено, если выполнено уравнение на Фурье-образ $\tilde{\varphi}(k, t)$ :

$$
\frac{d \tilde{\varphi}(k, t)}{d t}=w_{0} \Gamma_{p}(-\alpha)|k|_{p}^{\alpha} \tilde{\varphi}(k, t)+w_{0} \Omega\left(|k|_{p}\right)\left[\frac{1-p^{-1}}{p^{\alpha}-1}-\Gamma_{p}(-\alpha)|k|_{p}^{\alpha}\right] .
$$

С учетом того, что $\tilde{\varphi}(k, 0)=0$, получаем решение

$$
\tilde{\varphi}(k, t)=\Omega\left(|k|_{p}\right)\left(\frac{1-p^{-1}}{1-p^{-\alpha-1}}|k|_{p}^{-\alpha}-1\right)\left(1-\exp \left[w_{0} \Gamma_{p}(-\alpha)|k|_{p}^{\alpha} t\right]\right) .
$$

Поскольку $s(t)=\int_{Q_{p}} f(x, t) d_{p} x=\int_{Q_{p}} \tilde{f}(k, t) d_{p} k$ и $\tilde{f}(k, t)=\Omega\left(|k|_{p}\right)+\tilde{\varphi}(k, t)$, то

$$
\begin{gathered}
s(t)=1+\int_{Q_{p}} \tilde{\varphi}(k, t) d_{p} k= \\
=\frac{\left(1-p^{-1}\right)^{2}}{1-p^{-\alpha-1}} \sum_{i=0}^{\infty} p^{i(\alpha-1)}\left(1-\exp \left[w_{0} \Gamma_{p}(-\alpha) p^{-i \alpha} t\right]\right)+\left(1-p^{-1}\right) \sum_{i=0}^{\infty} p^{-i} \exp \left[w_{0} \Gamma_{p}(-\alpha) p^{-i \alpha} t\right] .
\end{gathered}
$$

Можно показать, что при $\alpha>1$ асимптотическое поведение данного ряда при $t \rightarrow \infty$ имеет вид

$$
\begin{aligned}
& s(t) \sim \frac{\left(1-p^{-1}\right)^{2}}{1-p^{-\alpha-1}} \frac{\left(-w_{0} \Gamma_{p}(-\alpha)\right)^{\frac{\alpha-1}{\alpha}}}{\alpha \ln p} t^{\frac{\alpha-1}{\alpha}} \sum_{k=-\infty}^{+\infty} \frac{1}{\left(\frac{\alpha-1}{\alpha}\right)^{2}+\left(\frac{2 \pi k}{\alpha \ln }\right)^{2}} \times \\
& \times\left(\left(\frac{\alpha-1}{\alpha} a_{k}-\frac{2 \pi k}{\alpha \ln p} b_{k}\right) \cos \left(\frac{-2 \pi k}{\alpha \ln p} \ln \left(-w_{0} \Gamma_{p}(-\alpha) \tau\right)\right)+\right. \\
& +\left(\frac{\alpha-1}{\alpha} b_{k}+\frac{2 \pi k}{\alpha \ln p} a_{k}\right) \sin \left(\frac{-2 \pi k}{\alpha \ln p} \ln \left(-w_{0} \Gamma_{p}(-\alpha) \tau\right)\right),
\end{aligned}
$$

где $a_{k}=\operatorname{Re} \Gamma\left(\frac{1}{\alpha}-\frac{2 \pi i k}{\alpha \ln p}\right), b_{k}=\operatorname{Im} \Gamma\left(\frac{1}{\alpha}-\frac{2 \pi i k}{\alpha \ln p}\right), \Gamma(x)-$ гамма-функция.

В силу (5) можно сделать вывод, что в предложенной модели асимптотическое поведение логарифмов цены актива при больших временах, предшествующих обвалу рынка, в точности описывается степенным законом с показателем $\frac{\alpha-1}{\alpha}<1$, умноженным на сумму логопериодических гармоник. Замечательным является факт, что этот показатель степени определяется только параметром $\alpha$, который определяет степень активности агентов и не зависит от параметра $p$, который есть отношение числа агентов соседних уровней иерархии.

\section{БИБЛИОГРАФИЧЕСКИЙ СПИСОК}

1. Сорнетте Д. Как предсказывать крахи финансовых рынков: критические события в комплексных финансовых системах. М.: Интернет-трейдинг, 2003. 400 с.

2. Metzler R., Klafter J., Jortner J. Hierarchies and logarithmic oscillations in the temporal relaxation patterns of proteins and other complex system / Proc. Natl. Acad. Sci. USA. V. 96. P. 11085. 1999. Chemistry.

3. Sornette D., Johansen A. A hierarchical model of financial crashes // Physica 261A, 1998, 581-598.

4. Владимиров В.С., Волович И.В., Зеленов Е.И. р-адический анализ и математическая физика. М.: Физматлит, $1994,352 \mathrm{c}$. 
5. Avetisov V.A., Bikulov A.Kh., Kozyrev S.V., Osipov V.A. p-Adic Models of Ultrametric Diffusion Constrained by Hierarchical Energy Landscapes // J.Phys. A: Math. Gen., 35 (2002) 177.

6. Avetisov V.A., Bikulov A.Kh., Kozyrev S.V., Application of p-adic analysis to models of spontaneous breaking of replica symmetry // Journal of Physics A, 1999. V.32. P. 8785.

7. Avetisov V.A., Bikulov A.Kh., Osipov V.A. p-adic description of characteristic relaxation in complex systems // J. Phys. A: Math. Gen. 36. 2003. 4239.

Поступила 15.01.2006 2.

УДК 517.958: 532.546

А.P. Самборук

МОДЕЛИРОВАНИЕ РАБОТЫ ГАЗОГЕНЕРАТОРОВ ФИЛЬТРАЦИОННОГО ТИПА

Рассмотрена математическая модель работы фильтрационного газогенератора, основанная на предположении о конвективном переносе тепла, обусловленного поступательным движением слоя реакиионноспособного расплава продуктов горения под действием перепада давления выделившихся газов.

Единственным способом, позволяющим получать газообразные продукты с температурой, близкой к начальной температуре заряда, является режим спутной фильтрации продуктов горения через несгоревшую часть пористого заряда [1-3].

Такой режим работы реализуется в газогенераторах фильтрационного типа [4-6]. Наиболее значительное влияние на параметры работы подобных газогенерирующих устройств (ГГУ) оказывает величина пористости газопроницаемого заряда и фазовый состав продуктов горения, образующихся при горении газогенерирующего состава (ГГС). Именно эти параметры определяют механизм передачи тепла и условия фильтрации продуктов горения через несгоревшую часть заряда.

Ранее уже были рассмотрены математические модели газовыделения с высокой и средней пористостью заряда с учетом и без учета фильтрационных затруднений [7-9].

Результаты анализа этих моделей фильтрационных генераторов, основанных на предположении о кондуктивном механизме передачи тепла в волне горения, показали, что характер изменения основных характеристик такого газогенератора (распределения давления по длине заряда, изменения скорости горения и давления в продуктах, а также максимального давления) не соответствуют экспериментальным данным. Поэтому была предложена совершенно иная модель горения заряда в фильтрационном газогенераторе, основанная на предположении о конвективном переносе тепла. Причем конвективный перенос тепла, прежде всего, связан с поступательным движением слоя реакционноспособного расплава легкоплавкого продукта разложения одного из исходных реагентов под действием перепада давления выделяющегося газа перед - и за слоем расплава. Для существования такого режима необходимо выполнение следующего условия: скорость тепловыделения за счет экзотермической реакции взаимодействия расплава с исходной шихтой должна быть выше скорости отвода тепла от расплава в непрогретую шихту. Физически это означает, что при попадании расплава в непрогретую шихту не происходит его застывания. Фильтрующийся инертный газ не может обеспечить опережающий прогрев исходной шихты, поэтому не может быть причиной реализации конвективного режима горения газогенерирующего заряда.

Рассматривается следующая модель газогенератора, изображенная на рис. 1 .

В трубчатый газогенератор, закрытый с одного из торцов газонепроницаемой крышкой, помещается исходная шихта с плотностью $\rho_{0}$ и пористостью $m$. Протяженность области, занятой шихтой, равна $l$, а свободного объёма $-l_{0}$. Инициирование процесса горения производится со стороны крышки, после чего по исходной смеси распространяется фронт реакции. Считается, что в результате взаимодействия исходных реагентов выделяется тепло ( $Q$ - на грамм смеси) и образуются конденсированные продукты (1-v грамм на грамм смеси), создающие неподвижный пористый каркас с пористостью $m_{1}$, а также инертный газ ( $v$ грамм на грамм смеси). 